\title{
The Astrophysical Plasma Emission Database: Progress and Plans
}

\author{
Randall K. Smith and Nancy S. Brickhouse \\ Harvard-Smithsonian Center for Astrophysics, 60 Garden St., \\ Cambridge, MA 02138 \\ Duane A. Liedahl \\ Department of Physics and Advanced Technologies, Lawrence Livermore \\ National Laboratory, P.O. Box 808, L-41, Livermore, CA 94550
}

\begin{abstract}
The Astrophysical Plasma Emission Database (APED) contains atomic data for the 14 most abundant astrophysical elements, collected from the literature. Although APED was originally designed for use in calculating a collisional-equilibrium X-ray spectrum suitable for analysis of high-resolution data, it is in a general format which can be efficiently used to calculate absorption spectra, photoionization models, and non-equilibrium collisional models. We emphasize original sources; each transition, rate, and energy level in APED contains a bibliographic reference. The APED can be downloaded from http://cxc.harvard.edu/atomdb/, or our website WebGUIDE

(http://obsvis.harvard.edu/WebGUIDE/) can be used to search for individual lines or transitions. We are continually working to expand APED (current version 1.3.1) and regularly issue updated collections.
\end{abstract}

\section{Introduction}

The goal of the Astrophysical Plasma Emission Database (APED) is to organize accurate and complete atomic data in a well-defined format that includes errors, original references, and version information as part of the electronically-available data. APED is used by a number of packages, including the Astrophysical Plasma Emission Code (APEC), which calculates the emission from a hot $\left(10^{4}-\right.$ $10^{9} \mathrm{~K}$ ) plasma, WebGUIDE (http://obsvis.harvard.edu/WebGUIDE/, a web tool that creates line lists and displays data from APED, and PHASE, a photoabsorption code (Krongold et al. 2003).

APED was designed to address the needs of the high-resolution X-ray data coming from the Chandra and XMM/Newton grating spectrometers, and so focuses on the hydrogen-like, helium-like, and iron and nickel L-shell lines that form most of the X-ray emission. The data are taken from the literature (e.g. Sampson, Goett \& Clark 1983, Jacobs et al. 1989) when available and from unpublished calculations if necessary. Some data for longer-wavelength lines was included from the CHIANTI 2.0 database as well (Landi et al. 1999)

When distributed, we combine the APED with the tables of calculated emission lines and continuum output from APEC; together, these form the atomic database ATOMDB, available at http://cxc.harvard.edu/ATOMDB. This site 
also contains documentation, software that can be used to access the ATOMDB, and any known caveats or issues with using the data.

\section{Future Plans}

Currently, APED includes a number of different ionization balance calculations. We plan to add ionization and recombination data so that APEC can calculate an equilibrium or non-equilibrium ionization balance as needed. In addition, we plan to add more inner-shell excitation/autoionization transitions, improve wavelengths for L-shell ions of carbon through calcium (based on lab data, e.g. Lepson et al. 2003), and add more dielectonic recombination lines.

\section{AstroAtom}

In creating APED, we have discovered that there is a gulf between atomic physicists and astrophysicists; frequently data exist in the atomic literature but are difficult for an outsider to discover. We have therefore begun a new website called "AstroAtom" (http://cfa-www.harvard.edu/astroatom/), which is designed to foster communication between atomic physicists and astrophysicists. The website is a joint venture of the Chandra X-ray Center and the Institute for Theoretical Atomic and Molecular Physics, and allows scientists to post abstracts, conference announcements, and questions in a search-able database.

\section{Acknowledgments}

The author acknowledges APED collaborators John Raymond and Priyamvada Desa. Support for this project is provided by NASA NAS8-39083 to SAO for the CXC and LTSA NAG5-3559.

\section{References}

Krongold, Y., Nicastro, F., Brickhouse, N.S., Elvis M., Liedahl,D. A. \& Mathur, S. 2003, ApJ, 597, 832

Sampson, D. H., Goett, S. J., \& Clark, R. E. H. 1983, ADNDT, 29, 467

Jacobs, V. L., Doschek, G. A., Seely, J. F., \& Cowan, R. D. 1989, Phys. Rev. A, 39, 2411

Landi, E., Landini, M., Dere, K. P., Young, P. R., \& Mason, H. E. 1999, A\&AS, 135, 339

Lepson, J. K., Beiersdorfer, P., Behar, E., \& Kahn, S. M. 2003, ApJ, 590, 604 